\title{
School Health Change Programs and Social Marketing, Does It Go Together?
}

\author{
Adamina Korwin-Szymanowska \\ Academy of Special Education, Poland
}

\begin{abstract}
The concept of health has generated a great deal of heated debate throughout the centuries. Its complexity embraces reciprocal relations between individuals and their social, financial, and cultural functioning. It is essential to focus on positive health promotion as it is a dominant factor contributing to the socio-economic development. School health programs appear to play a vital role in health competencies acquisition. They lay emphasis on health education by creating space for development of adaptive competence. This article provides an overview of framework for the process of developing school health programs by adapting principles of social marketing to requirements and health needs of target groups. Moreover, it demonstrates a background for health programs implementation aimed to convince audiences to adapt suggested patterns of behavior.
\end{abstract}

\section{Introduction}

Profound socio-economic changes of the past decades have led to the development of global knowledge society, where knowledge has become a key force used to meet the demands of today's world complexity. This shift has influenced education, which requires a new approach towards acquisition of competences essential in the 21 st century. One of the basic goals of education is to develop the socalled adaptive competences, understood by de Corte as the ability to apply gained skills and intentionally learned knowledge in everyday life. However, the core of this concept depends on an individual's health as a key factor for an overall personal and socio-economic development. Humans are unable to fulfill their educational potential without good health. As a result, this factor must be taken into account in the process of education planning. Improving one's health is a prerequisite for acquiring knowledge, therefore it is essential to focus on health education as a source of health improvement. Among various opportunities, school health programs appear to be a good solution to promote pro-health behavior.

The article focuses predominantly on the role of social marketing in school health programs as it paves the way for proper development of school health projects. Before providing a framework for action, the concept of health and social marketing must be introduced.

\section{In Search of Health's Nature}

At present the description of health and disease cannot be made in a monodisciplinary approach. Their understanding requires an integral system of interpretations which may be influenced by distractions caused by various factors. The diversity of health perspectives contributes to the ambiguity of this term which seems to be substituted by the concept of well-being, quality of life, or happiness [1] [2]. Moreover, the representations of phenomena closely related to health such as health behavior, lifestyle and health attitudes are also problematic to define.

Nevertheless, they pertain to health sciences. Therefore, they should be taken into account while reflecting on health. Despite the difficulties in formulating a universal definition of health, it has been found that health should be differentiated from disease. Secondly, it is associated with a state of welfare, high quality of life, as well as potential and capabilities which imply the positive context of interpreting. Moreover, as noted above, health is analyzed in multidimensional perspective, which may influence the methodological background of its defying. Over the years, modifications have been made to the definitions of health. Their number has grown to more than 300 [2].

One of the most famous definitions of health has been proposed by the World Health Organization. It was based on the Henry Sigerist's thesis, which assumes that human health consists of the internal balance between individual's body and mind, as well as its external relationship and interactions with the physical and social environment. A state of a perfect health is interpreted by Sigerist as a process of having control over individual's mental and physical capabilities, as well as having skills to adapt easily to changes observed in the environment. It would not interfere with possessed human resources and in the same time would contribute to the development of the society. He does not identify health with the absence of disease but in the context of a positive attitude towards life and responsibility imposed on every human being. 
The WHO applies a similar perspective in thinking about health, defining it as 'a state of complete physical, mental and social well-being and not merely the absence of disease or infirmity' [3]. This concise definition of health is considered to be a breakthrough in Health Sciences. It tackles not only the objective interpretation of health, but focuses predominantly on those aspects of health that appear to be, to a great extent, relevant to its psychological and social representations. Apart from the biomedical factors it pays attention to emotional and environmental functioning. Such an approach provides a shift from the medical interpretation towards the holistic model of health.

Health is a phenomenon entangled socially, culturally and economically, which refers to its complex nature. A holistic look at this issue expresses humanistic aspirations and pursuits. Nonetheless, many researchers considered the WHO definition as vague and utopian. Some researchers put arguments against the above mentioned explanation as they believe that the WHO definition treats health as a state of perfection, which is impossible to achieve for a vast majority of people. In addition, the WHO interpretation is nowadays criticized for lack of ecological aspect. However, both authors should realize that the concept of ecology was not popular in late 40s, thus it had not been included in the overall understanding of health at that time.

Moreover, interpreting health in the context of well-being reveals problematic concerns with operationalization of measurement indicators because of their ambiguity. The notion of well-being is usually associated with the category of the individual who is the only source being able to assess the subjective health. Given the above stated facts, the definition of the WHO is considered to be a very good starting point in constructing a positive definition of health.

The emergence of a new positive attitude towards health can be derived from a conviction that definitions based on static meaning are not fully convincing both for doctors and health educators. Our perception of health is always relative to our experience of its losing, regaining or keeping in shape. It indicates a weak relationship between the individual's activity towards maintaining health. As a consequence, it provides a good motivation for practicing medical activity focused on destroying symptoms of disease rather than its preventing. This pathogenetic health paradigm concentrates on finding the causes of disorders and curing the illness because it defines health as a lack of disease. Logically speaking, it is not possible to define the meaning of a word by negation, therefore such an interpretation cannot be fully accepted.

Apart from the above discussed problems stemming from the biomedical model of health, this paradigm focuses on the assumption that the disease has its etiological background generated by specific factors that lead to changes in structure and functioning of organism. Additionally, health is treated as a biological fact, which does not take into account social and environmental factors. Above all, the biomedical paradigm adopts a Cartesian assumption of separation of human soul and body, which is the essence of his dualism theory [1]. As a result, it compares the human body with a machine and all limbs and organs to parts of a machine which can be easily repaired or replaced if broken down.

Sęk and Heszen conclude that the above discussed model characterizes health as a lack of biological dysfunctions [1]. Body functions are normalized by medical standards based on biochemical processes of cells and organ functions are precisely measured by means of computerized methods for analyzing biological material. To summarize, in this model health and illness are disjoint categories, which leads to a conviction that an individual has no impact on his health. It reflects both a lack of adequate preventive care, as well as education that promotes a certain lifestyle and specific health behaviors.

While the above discussed biomedical paradigm was popular in medical sciences of the twentieth century, it has recently shown its limitations. The question which led to the development of a new health orientation is why a group of people exposed to the same health factors not always react in a similar manner to specific stimuli [4]. On the basis of these phenomena, Allen states that the biomedical model of health explains the disease at a cellular processes level [4]. Moreover, it presents health in a univariate way aggrandizing bio factor and ignoring the other aspects relevant to the functioning of an individual. According to Allen, the biomedical model focuses on treating disease rather than promoting health, which omits a significant aspect of multidimensionality [4].

Nowadays, the biomedical approach is not sufficient because of inefficient health care systems. The dynamism, positive attitude and individual's action must be taken into account in order to prevent a state of illness. The positive health definitions demonstrate the impact of human behavior, human responsibility, as well as all individual's features and characteristics, which constitute particular attitudes towards health. The concept of positive health is usually interrelated with the quality of life and individual's potential, which consist of selffulfillment and the ability to live and work actively. The positive health is linked to the overall development rather than just simply coping with difficulties in life. As a result, considerations associated with positive health go far beyond the traditional interest of medicine, which is based on 
health protection and restoration. It represents a key point in the philosophy of health promotion.

Skepticism associated with the above presented definition may be expressed due to lack of logical correctness and the ambiguity used in the term explained. In addition, many authors emphasize the need to incorporate the noetic aspect of health understanding in its definition which leads to conceptualization of health as a physical, psychological and social dimension unity. It imposes an outlook in which we interpret health as a holistic concept tied into existential significance based on a human activity, personal needs and aspirations, as well as a high quality of life, life energy, potential, development, ability to lead an active and productive life, enjoyment, optimism and happiness [2].

As a consequence, a new paradigm has appeared. Its assumptions refer to a man as a biological, psychological and social unity, hence it is called the biopsychosocial model of health As stated by Suls and Rothman, 'This perspective holds to the idea that biological, psychological, and social processes are integrally and interactively involved in physical health and illness [5]. The initially provocative premise that people's psychological experiences and social behaviors are reciprocally related to biological processes has fueled dramatic advances in health psychology over the past 25 years. Moreover, the premise that these subsystems are nested and inextricably connected has stimulated innovations in the design and implementation in interventions to promote health. As a guiding framework, the biopsychosocial model has proven remarkably successful as it has enabled health psychologists to be at the forefront of efforts to forge a multilevel, multisystem approach to human functioning [6].

A basic thesis of this model involves the interaction between the mind and the body, as well as the environmental and social influences, which affects many aspects of health and disease [4]. In contrast to the biomedical orientation, the biopsychosocial model treats an individual as a unity emphasizing the fact that apart from the disease there are other factors as motivation to recover, understanding the meaning of the disease and the social support which may affect the ability to recover [7].

The biopsychosocial model has at least two advantages. Firstly, it includes not only biological factors but also psychological and social ones, and secondly it views health in terms of positive definitions. Moreover, the biopsychosocial orientation is also perceived as a holistic and functional model of health.

While the former refers to the structure of the model, the latter focuses on functions of maintaining the internal equilibrium and the effective process of adaptation to requirements of the external environment. The holistic and functional model of health can be interpreted in the context of the ongoing relationship between a human being and their environment [1].

The process of a mutual adaptation leading to health has its footing in balancing the needs of individual and requirements of the environment. The level of health depends on the balance between pathogens and immune resources found in biological and mental characteristics of the individual and the social and cultural environment. According to Heszen and Sęk, an individual is a unit able to adapt to changing environmental conditions who is aware of the need of countervailing their own potential and external possibilities [1]. As a result, health is primarily understood as a process of adaptation aiming to maintain a dynamic balance between systems which depends on the individual's activity.

There have been many descriptive approaches towards human health. Among various paradigms proposed to specify health cognition, the most prominent has been depicted in the context of ecosystems, which gained a broad acceptance as a conceptual construct in the world we live in. As summarized by VanLeeuwen: 'Ecosystem health has been introduced as a paradigm for dealing with the interconnectedness of many global problems and complexities of managing and caring for our world' [8]. The tendency to interpret health in that framework resulted in the emergence of the Ecological Model, or the so-called Health Triad. It is based on a dynamic equilibrium between the host, the environment and the agent. Upsetting of the balance usually causes a disease. Further exploration shed a new light on the problem of health. In 1975 Morris introduced the Socioecological Model of Health. Although it focuses on disease instead of health and the influence of genetic and experiential host factors, as well as physical, social and environmental aspects, it has provided valuable contribution to health sciences.

In the middle of 1970 s the discussion shifted towards holistic models of health, which includes environment, lifestyle, biological nature and the system of health care [9] [10]. According to Lalonde, the Canadian Minister of National Health and Welfare, lifestyle has the greatest impact on human health because it determines its state in 50\% [10]. The Blum's model focuses on promoting health while taking into account the factors of population, culture, mental health, natural resources, and ecological balance [9].

The above reviewed models outlined the foundations of the Health Promotion development. The framework contributed to the appearance of a Mandala of Health advanced by Hancock and Perkins [11]. The Mandala of Health encompasses various dimensions which may influence the state of a human health: social and physical environments, culture, history, family, community services and 
infrastructure such as transport, schools and hospitals. Such an approach allows for concentrating not only on the human being as such, but also on surroundings. The Ottawa Charter for Health Promotion presented five action areas to provide socially and environmentally positive background for the development of health:

1. Building healthy public policy;

2. Creating supportive environments;

3. Strengthening community action;

4. Developing personal skills;

5. Reorienting health services.

Taking into consideration the above-discussed theoretical models related to health, it still remains an open question how to satisfactorily interpret health and disease, which are not only difficult to define, but even to think about. Health persistently escapes conscious observation efforts. Its subjective perception usually appears in the moment of disease appearance. However, the person's physical, psychological, and sociological functioning in a variety of contexts should be supplemented with a personal ability to adapt to internal disintegration of the personal's health system structural components, as well as an environment in which a man function.

Although the nature of health has been an ongoing puzzle consisting of many pieces, it is hard to match them in a gestalt reflecting all its aspects and dimensions. However, without making such attempts, the science will not arrive at any conclusions. That is why in health promotion it is necessary to reflect not only on the external factors, which influence health, but also on the most effective methods to achieve health behavior change.

A largely related phenomenon that has been studied extensively in psychology for a few decades concerns health behavior. As the research literature grows, it allows to distinguish many terms linked with health behavior such as medical behavior, health-related activities, health practices, illness behavior, or health-damaging behavior. Gochman established a health behavior's definition as 'those personal attributes such as beliefs, expectations, motives, values, perceptions, and other cognitive elements; personality characteristics, including affective and emotional states and traits, and overt behavior patterns, actions and habits that relate to health maintenance, to health restoration and to health improvement [12].

According to Gochman, health behavior may be analyzed in three ways: as risk behavior, as targets for change, or as interesting in their own rights [12]. An analysis of the above-mentioned categories enables us to make use of knowledge about health behavior in the context of school health change programs based both on theories and models of human behavior as well as behavioral change from both the individual and societal point of view. Given the fact that the literature provides rich theoretical insights and evidence in this matter, key models and ways of thinking about health behavior and its change are slightly mentioned. Evaluation of behavior change theory focuses on a distinction between models of behavior and theories of change, which seem to be complementary. Whereas the former identifies underlying factors of a specific behavior, the latter presents their change over time. As a result, the literature provides a vast range of theories and models, which may be applied. The authors reviewed several key constructs, however, for the purpose of this paper only selected examples are introduced.

One of the most common behavior models at the individual level is the Theory of Planned Behavior (TPB), which adopts a cognitive approach in explaining behavior, concentrating on personal attitudes and beliefs. It emerged from the theory of reason action, which considered the intention of act as the best predictor of behavior. Ajzen asserts that 'the theory of planned behavior is based on the assumption that human beings usually behave in a sensible manner; that they take account of available information and implicitly or explicitly consider the implications of their actions. Consistent with this assumption (...), the theory postulates that a person's intention to perform (or not to perform) a behavior is the most important immediate determinant of the action' [13].

From this perspective, intentions are determined by three factors: the personal factor, which is described by the agent's attitude towards the behavior, the individual's perception of social influence and pressure to perform or not termed subjective norm, and thirdly, perceived behavioral control, which is grounded on the sense of selfefficacy and potential to perform the particular behavior. Taking into account the above mentioned factors, people usually tend to demonstrate a behavior when a specific combination of positive evaluation appears. It is associated with expected outcomes, experience of social pressure and conviction of possessing the right means and opportunities to fulfill the behavior.

The second individual theory based on the assumption that behavior is determined by numerous beliefs concerning threats to a personal well-being, as well as the effectiveness and outcomes of actions, is termed the Health Belief Model (HBM). This theoretical construct presents the significance of cognitive-evaluative structures of the individual in explaining the behavior orientated towards health. The author adds that the whole concept is based on an assumption that decision concerning the pursue a particular activity is a consequence of the individual's assessment of behavioral usefulness of the results. A perceived threat is the core of the HBM 
because it is associated with the individual's readiness to take action. This leads to a subjective perception of susceptibility and vulnerability to a specific risk and the seriousness of potential consequences derived from taking new behavior. The person needs to establish the extent to which his behavior is beneficial in order to change it. Barriers perceived by the individual, as well as perceived self-efficacy are the most important determinants of behavior. Notwithstanding, the analyzed phenomenon requires a presence of motivation interpreted from both cognitive and emotional perspectives.

Yet another common conceptualization of change behavior, firstly introduced in relation to smoking, is known as Stages of Change labeled also as a Transtheoretical Model. It assesses a personal readiness to start a new healthy behavior. It views the behavioral change as a continuum process which consists of five stages: precontemplation, contemplation, preparation, action, and maintenance. It focuses predominantly on the transition between consecutive steps driven by self-efficacy and the assessment of advantages and disadvantages of behavioral outcomes. Although its aim is to maintain the course of change, it is popular to move backwards through the stages. That situation may not fully cover the need of change, especially while considering smoking addiction. What is more, the model depicts the role of the individual underspecifying the social, economic, and environmental factors which are located outside the "self". Consequently, it has been widely criticized for its understatements and the lack of clarity.

The socio-psychological models of individual's behavior tend to evoke to a decision making process, which is consistent with the economic theories that most of the people are likely to behave rationally, enlarging their profits. However, another theory has emerged in order to fill the scientific gap referring to multidimensional perspectives of behavior. The Social Practice Theory (SPT) as one of the sociotechnical systems, is an approach encompassing diversified interrelated components, such as physical and mental activity, knowledge, norms, or meanings. It provides the framework for everyday human action. The environmental, socio-technical, and infrastructural factors play a vital role in constituting individual behavior. This thesis is supported by Shove, who implemented the three-element model composed of materials (physical objects which facilitate performing activities), meanings (interpretations, images, or concepts which appeal to the moment of performance), and procedures (which enable the subject to take action based on skills and competencies) [14]. However, he sums up that all models putting emphasis on integration of both individual and social perspectives are rather doomed to fail. Nevertheless, the frame of SPT presupposes a certain viewpoint on the possibility to complete various aspects of human functioning in a vast range of contexts.

The results of the above reviewed models provide evidence for endeavors taken in order to explain health behavior and to present various concepts of their change, as well as to demonstrate that they play an important role in structuring conceptualizations of the notion of health behavior. Although it still remains underspecified, it leaves open the possibility of different construals. However, the selected models may provide a considerable input in school health change programs.

\section{Understanding Social Marketing}

The concept of social marketing stems from an amalgamation of social sciences and marketing. The later has been defined in various ways, but for the purpose of this paper the interpretation of the American Marketing Association has been applied. It describes marketing as the activity, set of institutions, and processes for creating, communicating, delivering, and exchanging offerings that have value for customers, clients, partners, and society at large.

Social marketing may be considered as a branch of marketing, since both fields share a number of similarities. To begin with, they are customer orientated, because they refer to mutual relations with a target audience. Next, both disciplines are entangled in a process involving various stages implemented in order to achieve particular goals [15]. However, they are separated by a prevailing discrepancy grounded on the attitude towards the subject of marketing. As emphasized by Oldenburg and Parcel, while commercial marketers sell services and goods, social marketers are focused on selling positive behavior [16]. Additionally, while commercial marketing situates a product in relation to other goods by generating competitive relation between companies, social marketing exerts an impact on target group's behavior by appealing to future benefits. Moreover, public welfare is the quintessence of social marketing, whereas the key aim of commercial marketing is to multiply the wealth of shareholders [17]. Nevertheless, they both rely on marketing mix also labelled as four P's, which includes product, price, place, and promotion [16]. Social marketing may be then understood as: 'a process that applies marketing principles and techniques to create, communicate and deliver value in order to influence target audience behaviors that benefit society as well as the target audience' [18].

The above approach is widely used in public health, but it may be also implemented in school health programs. Argumentation for the use of social marketing principles for that purpose is based on an assumption that applying marketing methods in 
school health programs will increase the effectiveness of intended outcomes. Siegel and Lotenberg claim that: 'The principles of marketing provide a disciplined, audience-focused, researchbased process to plan develop, implement, and assess many different types of initiatives designed to improve the public's health or use and appreciate public health institutions' [19].

Consequently, because of the above-reviewed features, social marketing and its components may also be used in school health programs, which rely predominately on changing one's negative healthrelated behavior patterns through promotion of positive ones.

\section{Social Marketing Stages in School Health Programs}

In order to provide a proper development of school health programs, various stages included in the social marketing framework must be taken into account. Although the literature offers numerous examples of stages which may influence the process of health behavior change, this paper focuses on the proposition put forward by Kotler and Lee [15] [18].

Step 1. Defining a Problem: To begin with, Kotler and Lee refer to a precise definition of a problem, which is the subject of action [15]. Its defining is based on certain data and information obtained as an outcome of scientific research. A preliminary analysis of the problem requires an adequate background for project realization. It encompasses factors leading to the need of introducing health programs. However, education should rather anticipate certain health problems, therefore its main concern should be based on health promotion and risk prevention.

Step 2. Situation Analysis: The next stage includes a specification of benefits which should be generated by the school health program in a target group. This stage includes defining goals and describing available resources, which is used to maximize the impact of a school project and ensure its feasibility. In order to provide those components, it is essential to delineate the situation on the basis of SWOT analysis, which is a structured planning method used for evaluation of organizational strengths and weaknesses, as well as environmental opportunities and threats which may occur during the realization of a health project. Additionally, following guidelines for literature reviews may contribute to the achievement of intended objectives.

Step 3. Target Group: The following step describes a target audience selection. Taking into account pupils in a classroom, the process of segmentation should include not only the division of a group prone to potential health risk factors, but also the manner of acquiring knowledge. The program of the project should be accommodated to the needs and possibilities of the learners. In order to achieve the best outcomes, a recognition of multiple intelligence (Gardner) and implementation of adequate teaching techniques is a conditio sine qua non. It is noteworthy that effective health change must be simultaneously targeted at all relevant groups, namely children, parents, and teachers. The holistic approach towards health behavior change paves the way for project's success.

Step 4. Objectives: On the basis of the abovediscussed components, clear health program objectives may be established. As stated by Cheng, Kotler and Lee, 'a social marketing campaign needs clear marketing objectives and goals. Specifying desired behaviors and changes in knowledge, attitudes, and/or beliefs, marketing objectives always includes a behavior objective - something you want the target audience to do as a result of the campaign' [18]. Similar components must be taken into account in the development of school health programs. Distinguishing knowledge objective from belief objective ensures the comprehensive approach towards health education process, as it involves not only information to be acquired by the target group, but also change of health-related behavior, which reflects a modification of human mind structures. In order to provide comprehensive critical analysis of school health project's purposes, a SMART criteria should be followed. They delineate goals, which should be specific, measurable, achievable, relevant, and time-bound. The SMART strategy focuses predominantly on a particular area of improvement by ensuring measurable indicators of progress. Moreover, it outlines the way in which the established goals should be achieved in order to obtain desirable results in a particular time.

Step 5. Factors of Influence: The fifth stage concentrates on different components that may influence the target group behavior. Their analysis consists of identifying both obstacles and competitors, as well as potential benefits and influencers. It should take into account recognition of barriers that appear to be a significant part of the process, as well as pinpointing tangible benefits significant from learners' perspective. Therefore, identifying reasons which may prevent pupils from adopting promoted behavior may contribute to some specific dynamic qualities included in the project's frame. However, a fundamental issue in this case relates to benefits' exposition, which plays a key role in children's perception of the school health program. 
Step 6. Positioning Statement: A prominent feature of the sixth stage is based on delineation of a positioning statement, which labels emotions and benefits which should be felt about the target behavior by the students. The positioning statement is a foundation of marketing mix strategy.

Step 7. Marketing Mix Strategy: Marketing mix strategy consists of four instruments: product, price, place, and promotion. It has become a tool for constructing, communicating, and transferring values for the target behavior. An accurate explanation of a product is the main aim of that strategy. It is interpreted on three tiers: core, actual, and augmented. The first one outlines profits gained by pupils when the target behavior is implemented. Whereas the actual product is described as a wanted behavior displaying particular characteristics, the augmented product is interpreted as products, goods, or services which are additionally included in the health program. The next strategy refers to price understood in both financial and non-financial terms. It includes costs which have to be paid by school children in order to implement a target health behavior. Cheng, Kotler and Lee sum up that a wise health program tries to reduce costs in order to promote health behavior [18]. A place strategy device provides an area of action, which includes a place of performance, as well as other services, objects, and projects interrelated with the school health project. Promotion is the last component of the marketing mix strategy, which determines a fruitful communication. It encompasses a vast range of communication channels, essential messages and taglines, as well as their implementation.

However, Donovan introduces a new approach towards a marketing mix strategy, which presents social marketing aims with reference to Ottawa Charter on Health Promotion [20]. It is predominantly targeted at individuals in order to (1) inspire them to change their behavior, (2) to influence industrial producers to manufacture goods and services consumer's health friendly, (3) make changes in places in order to prevent people from risky behavior and promote healthy one, and (4) encourage people with political power to make decisions interrelated with pro-healthy perspective.

All the above-reviewed components of successful communication require customization to the audience. They should be adjusted to the participants, as school children vary according to numerous factors. Consequently, in order to provide the best impact of school health change programs, it is inevitable to analyze all established goals and barriers, as well as children's possibilities, which will lead to a communication success. Taking into account pupils, various channels of communication should be employed to augment the potential effects of the health project. Nowadays, there is a wide spectrum of possibilities, however, the best option is to select multidimensional tools, which would influence both right and left hemisphere, as it expands the process of learning and makes it more effective.

Step 8. Monitoring and Evaluation: Monitoring and evaluation is the next step, which is particularly vital in education and school health programs. Whereas the former is interpreted as a tool providing necessary enhancement during the project, the latter is defined as its final assessment. Evaluation is conducted on the basis of established aims. Kotler and Lee suggest some questions that appear to be helpful in the process of monitoring and evaluation, e.g. 'Why will this measurement be conducted? For whom? What inputs, processes, and outcomes/impacts will be measured? What methods (such as interview, focus group, survey, and/or online tracking) will be used for these measurement? When will these measurements be conducted? How much will these measurements cost?' [15].

Considering health issues, education is principally interested in learning outcomes emerging from the project, as well as sustaining its long-term effects, which still remains rather unsatisfactory in some aspects of education.

Step 9. Budget and Funding: Setting up a budget is the following step, which is perceived as another element significant from the school health program's perspective. This stage requires an analysis of costs, which must be integrated with aims and activities conducted during the whole process. However, ceaseless monitoring of correspondence between a planned budget and expenses is required in order to achieve the intended goals.

Step 10. Plan for School Health Program: Planning a school health project implementation and its management is the last stage culled from social marketing. It includes collecting exact information about people, time, and resources to be incorporated in the whole health change program. It provides a framework for action by emphasizing subsequent steps leading to the achievement of goals.

\section{Conclusions}

The above-reviewed model of social marketing highlights the conceptual correspondence between particular stages of social marketing campaigns' process and its influence on school health programs targeted at informing and persuading pupils to cease unhealthy patterns of behavior and adopt healthy ones. As a result, it leads to a social change, which strives for reducing discrepancy between different social inequalities. 
However, what makes marketing and social marketing, as well as school health programs particularly difficult is the exchange process. In marketing, the exchange process involves money paid for goods or services. Consequently, financial resources enable a client to make a desired transaction. By paying, the client expects a certain level of customer service. This psychological mechanism provides a balance between customer and seller's involvement in the whole exchange operation. This procedure may fail when social marketing is taken into account. Although it proposes valuable solutions to various health problems, most of people do not feel motivated to change their behavior as they are not fully engaged in the process. The perspective of putting effort in changing one's behavior may be solely balanced by perception of potential benefits.

One must bear in mind, however, that if the level of requirements interpreted in terms of health behavior change is too high, the whole process will fail. A step-by-step strategy appears to be a better solution as it does not change an individual as a subject to a great extent. When human integrity as a whole is not disturbed, small health changes in behavior patterns are more likely to occur.

Apart from the above discussed reasons, another issue significant from a school perspective is evaluation. Despite its importance, this component still causes problems. Unfortunately, some schools lag behind with long-term assessment of project's outcomes, which is a foundation for future actions and lessons learned.

Moreover, the core of health education lays in developing individual self-responsibility for health. Taking it into account will augment the effectiveness of learning at every stage of the educational process.

Finally, although social marketing and its principles may be perceived as a useful tool for school health programs, they should be supported by reflection on values and attitudes important from the educational and socio-cultural point of view.

\section{References}

[1] Heszen, I, H. Sęk, Psychologia zdrowia, Wydawnictwo Naukowe PWN, Warszawa, 2007.

[2] Woynarowska, B., Edukacja zdrowotna. Podręcznik akademicki, Wydawnictwo Naukowe PWN, Warszawa, 2007.

[3] WHO (World Health Organization) (1948). Preamble to the Constitution of the World Health Organization as adopted by the International Health Conference, New York, 19-22 June, 1946; signed on 22 July 1946 by the representatives of 61 States (Official Records of the World Health Organization, no. 2, p. 100) and entered into force on 7 April 1948; http://www.who.int/about/definition/ en/print.html (6 January 2013)
[4] Allen, F., Health Psychology: Theory and Practice, Allen \& Unwin Pty Ldt., Australia, 1998.

[5] G.L. Engel, "The Need for a New Medical Model: A Challenge for Biomedicine", Science 196, 1977, pp. 129136.

[6] J. Suls, A. Rothman, "Evolution of the Biopsychosocial Model: Prospects and Challenges for Health Psychology", Health Psychology, 23(2), 2004, pp. 119-125. doi:10.1037/0278-6133.23.2.119

[7] DiMatteo, M. R., L. R. Martin, Health Psychology. Allyn \& Bacon, 2002.

[8] J. A. VanLeeuwen, D. Waltner-Toews, T. Abernathy, B. Smitt, "Evolving Models of Human Health Toward an Ecosystem Context", Ecosystem Health, 5(3), 1999, pp. 204-219.

[9] Blum, H. I., Planning for Health: Development Application of Social Change Theory, New York: Human Sciences Press, New-York, 1974.

[10] Lalonde, M. (1974/1981) 'A new perspective on the health of Canadians'; http://www.phac-aspc.gc.ca/phsp/pdf/perspect-eng.pdf (5 January 2014)

[11] T. Hancock, F. Perkins, "The Mandala of Health: A conceptual model and teaching tool", Health Education, 24, 1985, pp. 8-10.

[12] Gochman, D. S., Labels, systems, and motives: Some perspectives for future research. In D. S. Gochman \& G. S. Parcel (Eds.), Children's health beliefs and health behaviors. Health Education Quarterly, 9,1982, pp. 167174.

[13] Ajzen, I., Attitudes, Personality and Behaviour., Oxford University Press, England, 2005.

[14] Shove, E. (2010) 'Submission To The House Of Lords Science And Technology Select Committee Call For Evidence On Behaviour Change'; http://www.Lancs.Ac.Uk/Staff/Shove/Transitionsinpractic e/Papers/Shovehouseoflords.Pdf (11 April 2014).

[15] Kotler, P., and N.R. Lee, Social Marketing. Influencing Behaviors for Good, 3rd Ed. Thousand Oaks, Sage Publications, USA, 2008.

[16] Oldenburg, B., and G. Parcel, Diffusion of innovations, in K. Glanz, B.K. Rimer, and F.M. Lewis (Eds.), Health Behavior and Health Education, Wiley, San Francisco, 2002.

[17] Kotler, P., N. Roberto, N. Lee, Social Marketing: Improving the Quality of Life, Sage Publications, Inc., USA, 2002, p. 20.

[18] Cheng, H., P. Kotler, and N. Lee, Social Marketing for Public Health. Global Trends and Success Stories, Jones and Bartlett Publishers, USA, 2011.

[19] Siegel, M., and L.D. Lotenberg, Marketing Public Health. Strategies to Promote Public Health, Jones and Bartlett Publishers, USA, 2007, p.203. 
International Journal of Technology and Inclusive Education (IJTIE), Volume 4, Issue 2, December 2015

[20] R.J. Donovan, The role of social marketing in public health change programs, Australian Review of Public Affairs, Vol.10, No. 1, July 2011, pp. 23-40. 